\title{
Identification and Determination of Rubrofusarin, Rubrofusarin Isomer, and Their Quinone Forms in Grains Using High-Resolution Mass Spectrometry
}

\author{
Song-shan Wang, ${ }^{\dagger}$ Hua Cui, ${ }^{\dagger}$ Jin Ye,,$^{\dagger} \mathrm{Yu} \mathrm{Wu}{ }^{\dagger}$ Song-xue Wang, ${ }^{*}{ }^{\dagger}$ and Wen-bing Yin ${ }^{\ddagger} \odot$ \\ ${ }^{\dagger}$ Academy of State Administration of Grain, 11 Baiwanzhuang Street, Xicheng District, Beijing 100037, People’s Republic of China \\ ${ }^{\ddagger}$ State Key Laboratory of Mycology, Institute of Microbiology, Chinese Academy of Sciences, Beijing 100101, People’s Republic of \\ China
}

Supporting Information

\begin{abstract}
Fungi of the genus Fusarium can produce secondary metabolites such as naphthopyrones and naphthoquinones that are toxic and expected to threaten the food and feed safety. In this study, the occurrence of rubrofusarin, rubrofusarin isomer, and their quinone forms in grains was identified and confirmed using ultrahigh-performance liquid chromatography coupled with hybrid quadrupole orbital ion trap mass spectrometry (Q-Orbitrap MS). The quantitation of these compounds in grain samples was also investigated using Q-Orbitrap MS. The results showed the concentrations of rubrofusarin ranged from 3.278 to $33.82 \mu \mathrm{g} / \mathrm{kg}$, from 0.815 to 61.86 $\mu \mathrm{g} / \mathrm{kg}$, and from 7.362 to $47.24 \mu \mathrm{g} / \mathrm{kg}$ for the maize, rice, and wheat samples, respectively. By comparison, the abundances of their quinone forms were relatively lower, and the concentration of quinone form of rubrofusarin isomer was relatively higher than that of quinone form of rubrofusarin. These compounds were also confirmed to coexist with other known Fusarium mycotoxins. The data-dependent tandem mass spectra obtained from the Q-Orbitrap MS were validated to provide a wealth of valuable information that allowed for advanced data interpretation for solid confirmation of these compounds in grains. To the best of our knowledge, this is the first study that concerns the occurrence and quantitation of rubrofusarin, rubrofusarin isomer, and their quinone forms in grains.
\end{abstract}

\section{INTRODUCTION}

The fungal genus Fusarium contains numerous species that can infect a wide variety of cereal crops in the field and cause plant diseases such as ear rot and head blight. ${ }^{1}$ Species of the genus Fusarium can produce a great number of toxic metabolites during crop growth and storage under specific climatic conditions. $^{2,3}$ These include the most common mycotoxins such as the trichothecenes, the fumonisins, and zearalenone (ZEN), for which regulatory limits and standard detection methods have been established in many countries. ${ }^{4-6}$ However, several other toxic metabolites produced by Fusarium spp. are expected to coexist with these well-investigated mycotoxins, and may bring a potential issue relating to food and feed safety. And, therefore, the identification of these unnoticed toxic metabolites in food and feed deserves some attention.

Ultrahigh-performance liquid chromatography (UHPLC) coupled with high-resolution mass spectrometry is one of the recently developed and powerful analytical approach that has been used for the detection of mycotoxins in a wide variety of matrices. ${ }^{7-10}$ Compared with the traditional instrumental methods like liquid chromatography coupled with ultraviolet, fluorescence detector and triple quadrupole mass spectrometry, ${ }^{11-13}$ one of its advantage is the use of full-scan acquisition mode, which allows for the retrospective analysis of samples for screening other nontargeted analytes besides the targeted mycotoxins. High-resolution mass spectrometry has proved to be effective in the tentative identification of a variety of potential risk substances in food and feed. ${ }^{14,15}$ And, therefore, it provides the possibility for screening the unnoticed fungal toxic metabolites in food and feed during the routine detection of mycotoxins.

Rubrofusarin and aurofusarin, produced by a variety of Fusarium spp., are naphthopyrones and naphthoquinones classes of polyketides that contain a variety of fungal and plant metabolites that have biological activities. ${ }^{16}$ Previous studies demonstrate that rubrofusarin has anti-estrogenic effects ${ }^{17}$ and can inhibit the activity of human DNA topoisomerase II- $\alpha{ }^{18}$ Aurofusarin is observed to be cytotoxic in mammalian cells ${ }^{19}$ and can generate oxidative stress and induce DNA damage. ${ }^{20}$ Therefore, rubrofusarin and aurofusarin are expected to contribute to the overall toxicity of respective Fusarium mycotoxin mixtures. Recently, it is reported that the occurrence of aurofusarin is prevalent in different types of cereal-based food and feed commodities. ${ }^{21-23}$ However, none of the studies

Received: August 17, 2018

Accepted: November 7, 2018

Published: November 27, 2018 

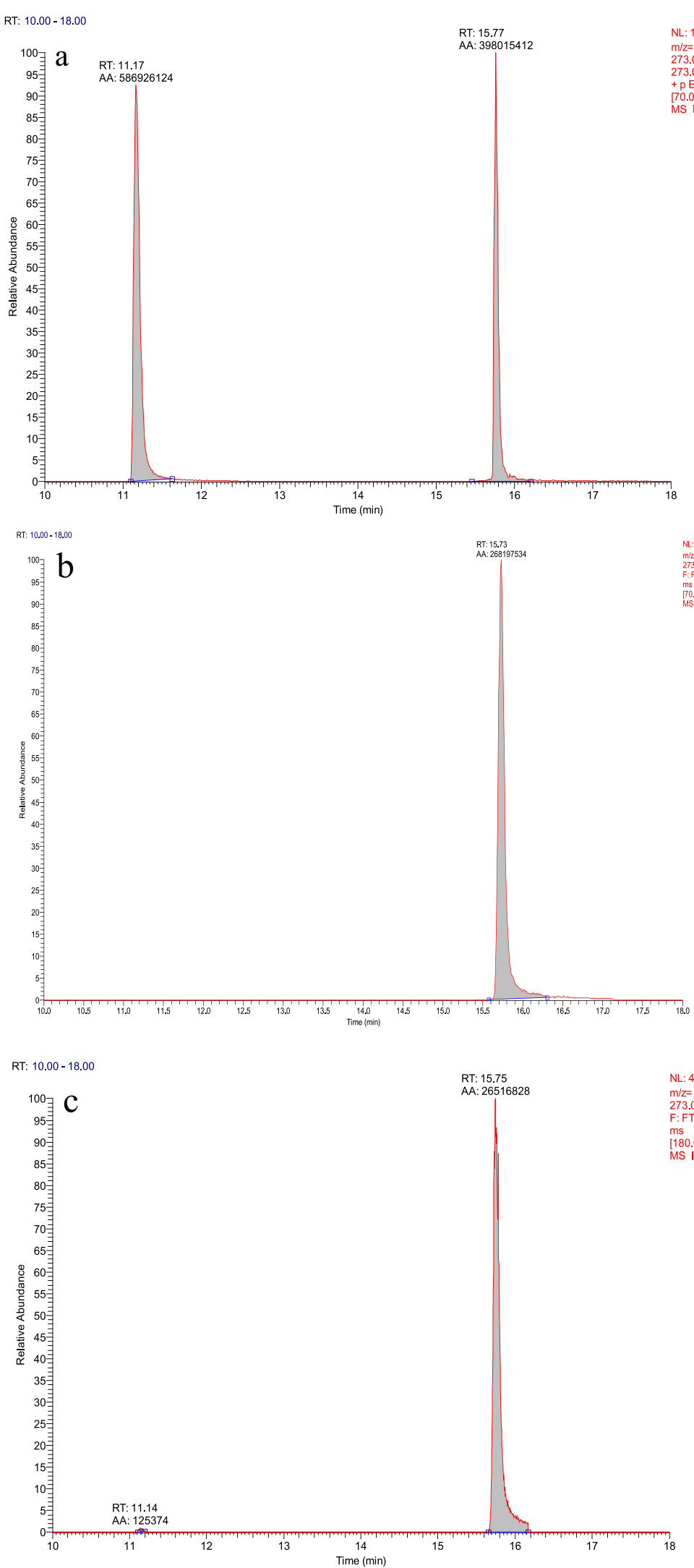

Figure 1. UHPLC/Q-Orbitrap MS analysis of rubrofusarin and its isomer in the metabolites of F. graminearum (a), rubrofusarin standard (b), and representative real grain sample (c, rice sample).

reported the occurrence of rubrofusarin in food and feed, as it is usually considered as one intermediate in fungal biosynthetic pathways (e.g., the aurofusarin pathways in Fusarium graminearum $)^{24}$ and cannot be accumulated during the growth of fungi. ${ }^{25}$ This precludes the comprehensive understanding on the potential risks of Fusarium mycotoxins in food and feed. 
In this study, the occurrence of rubrofusarin in grains was identified and confirmed using hybrid quadrupole orbital ion trap mass spectrometry (Q-Orbitrap MS). And together with rubrofusarin, its isomer and quinone forms were also found in grains. The quantitative and qualitative methods of these compounds were also investigated. These results indicated the co-occurrence of rubrofusarin, rubrofusarin isomer, and their quinone forms with the Fusarium mycotoxins like deoxynivalenol (DON) and zearalenone (ZEN) in grains.

\section{RESULTS AND DISCUSSION}

Rubrofusarin and Its Isomer Produced by F. graminearum. F. graminearum is one of the species of genus Fusarium that could produce rubrofusarin. ${ }^{26}$ Therefore, the metabolites of F. graminearum were first analyzed to investigate the production of rubrofusarin. Out of our expectation, the extracted ion chromatogram of $m / z 273.0757\left([\text { rubrofusarin }+\mathrm{H}]^{+}\right.$) revealed two ion peaks besides those of rubrofusarin (Figure 1a,b). The product ions of these two precursor ions were both identical with those of rubrofusarin standard (Figures S1a-c and S2), indicating one isomer of rubrofusarin could also be produced by F. graminearum. To the best of our knowledge, this is the first study to report the discovery of rubrofusarin isomer, and its structure is still unclear.

The production of rubrofusarin and its isomer during the growth of $F$. graminearum on maize matrix was further dynamically investigated. The yields of these two metabolites were expressed as the corresponding peak areas. The results showed an increasing accumulation of rubrofusarin throughout the 30 days of cultivation, whereas the production of rubrofusarin isomer reached the maximum after 10 days of cultivation and then decreased gradually to less than $10 \%$ of the maximum level (Figure 2). These results indicated that it was the

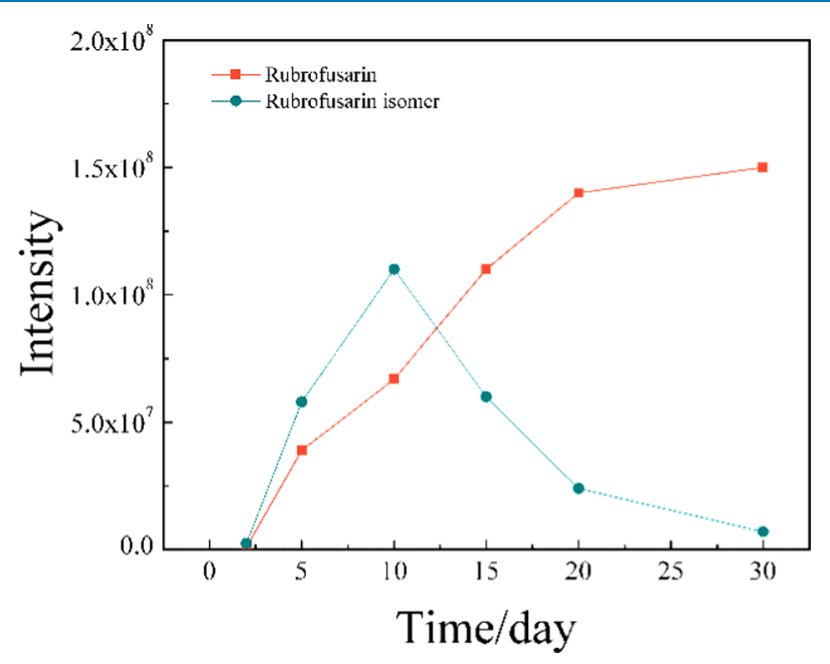

Figure 2. Production of rubrofusarin and its isomer by F. graminearum growing on maize matrix.

rubrofusarin isomer but not the known rubrofusarin that could not be accumulated during the growth of F. graminearum. The decline of rubrofusarin isomer might provide new insights into the biosynthesis of metabolites using rubrofusarin as an intermediate.

Occurrence of Rubrofusarin and Its Isomer in Grains. Because rubrofusarin could be accumulated during the laboratory culture of F. graminearum, it raised our concerns that whether rubrofusarin and its isomer existed in the real grain samples. The "dilute-and-shoot" sample preparation and full scan data acquisition using UHPLC/Q-Orbitrap MS had been previously validated and applied to the multianalysis of mycotoxins in grains in our previous studies. ${ }^{27}$ The advantage of this approach was to allow for the retrospective analysis of the samples for the screening of substances in the in-house database. And, therefore, we reprocessed the raw data of naturally contaminated grain samples using an in-house database containing rubrofusarin. The screening results showed the presence of rubrofusarin in grains, which could be confirmed by the well match of identification criteria (Figures 1c and S1d). Although, for rubrofusarin isomer, the weak ion peak indicated its presence, the abundance was too low to trigger a datadependent $\mathrm{MS}^{2}\left(\mathrm{ddMS}^{2}\right)$ scan to make a confirmation. The low abundance of rubrofusarin isomer in grains was possibly attributed to its decline during the growth of fungi, as discovered above.

Occurrence of Quinone Forms of Rubrofusarin and Its Isomer in Grains. As one type of naphthopyrone, rubrofusarin was expected to be oxidized to its quinone form with a formula of $\mathrm{C}_{15} \mathrm{H}_{10} \mathrm{O}_{6}$ and theoretical $\mathrm{m} / z$ of $287.0555\left([\mathrm{M}+\mathrm{H}]^{+}\right){ }^{25}$ To validate this, rubrofusarin standard was dissolved in methanol solution (methanol/water $=1: 1, \mathrm{v}: \mathrm{v}$ ) and then subject to oxidation by purging filtered air bubble in the solution. The solution was diluted and then subject to mass detection after 2 days oxidation. The extracted ion chromatogram revealed an ion peak with $m / z 287.0555\left([\mathrm{M}+\mathrm{H}]^{+}\right.$, Figure 3a), indicating the generation of quinone form of rubrofusarin. The major product ion had a formula of $\mathrm{C}_{13} \mathrm{H}_{8} \mathrm{O}_{5}(\mathrm{~m} / z$ 244.0370, Figure S3a), which was consistent with the quinone form of product ion from rubrofusarin $\left(\mathrm{C}_{13} \mathrm{H}_{10} \mathrm{O}_{4}, m / z 230.0574\right.$, Figure S1). These results confirmed that it was the oxidation product of rubrofusarin (quinone form). The oxidation of rubrofusarin could also be observed during the instrumental analysis due to the presence of oxygen in the extraction solution and mobile phase. And, therefore, it was possible to investigate the quinone form of rubrofusarin isomer in the cultures of $F$. graminearum. The extracted ion chromatogram of $\mathrm{m} / z 287.0555$ revealed three ion peaks (Figure $3 b$ ), and the fragment ions were all identical with those of quinone form of rubrofusarin, indicating they were the corresponding oxides (Figure $S 3 b-d$ ). The quinone form of rubrofusarin could not be separated from rubrofusarin isomer under the present chromatographic condition. This explained why three ion peaks but not four could be observed on the chromatogram.

A screening of quinone forms of rubrofusarin and its isomer was further carried out and the result confirmed their presence in the real grain samples (Figures $3 c$ and $S 3 e, f$ ). The relative intensity revealed a comparable abundance of these two quinone forms, which was contrary to the relative lower concentration of rubrofusarin isomer in grains, indicating it could be more easily converted to its quinone form compared with rubrofusarin.

Method Validation for the Quantitation of Rubrofusarin in Grains. The presence of rubrofusarin, rubrofusarin isomer, and their quinone forms raised an urgent concern on their contamination levels in grains. However, to date, no method has been reported for the quantitation of these compounds. Considering the wide applicability of the previously developed approach for a multianalysis of mycotoxins based on UHPLC/Q-Orbitrap MS, ${ }^{27}$ it was directly utilized for the quantitation of these compounds in grains. The method 

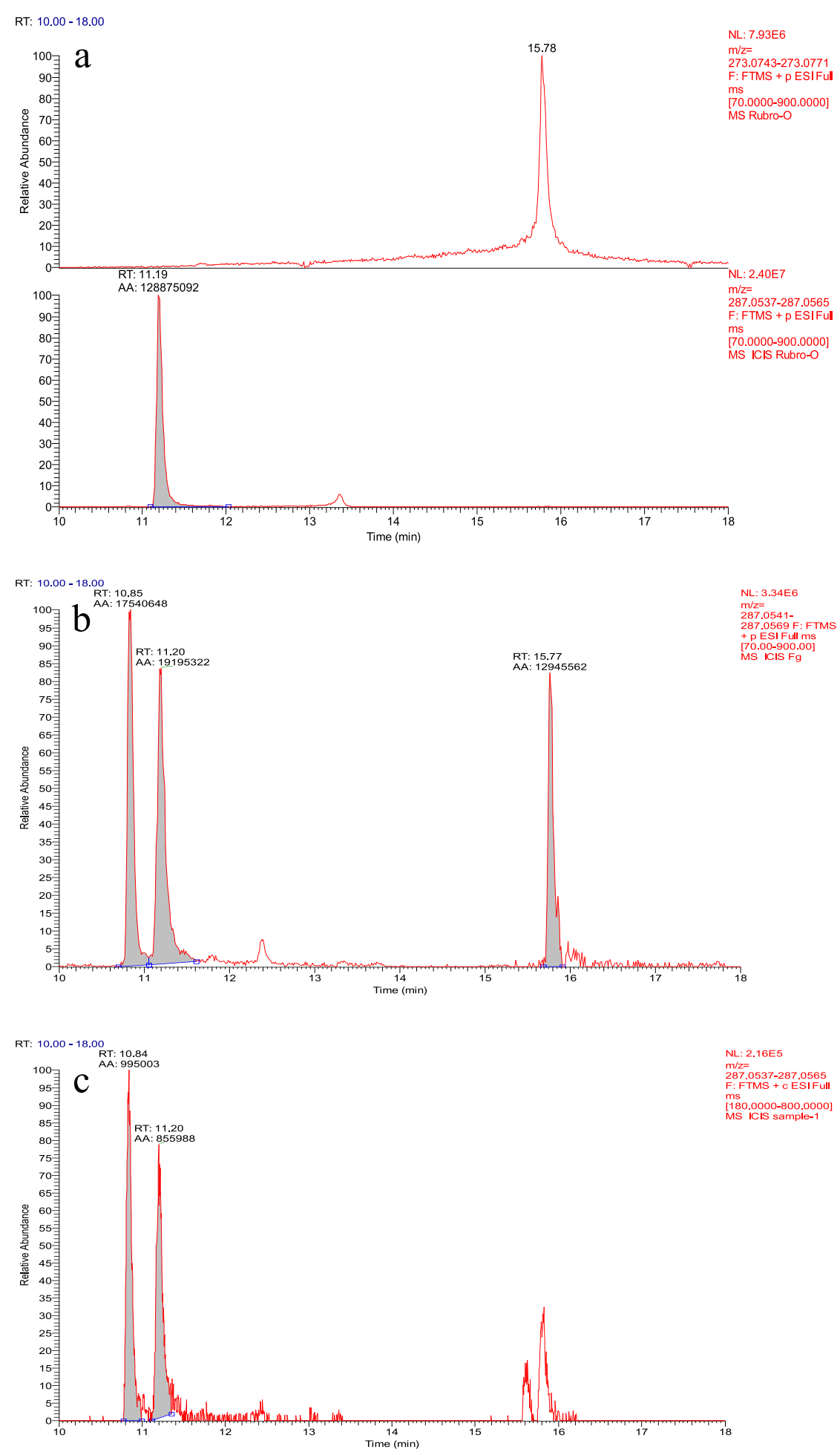

NL: 3.34E6

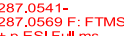

p ESIFEll ms
$70.00-900.00]$

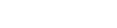


Table 1. Averaged Recoveries $(n=9)$ and Relative Standard Deviations (RSDs) for Rubrofusarin at Four Spiking Levels in Maize, Wheat, and Rice

\begin{tabular}{|c|c|c|c|c|c|c|}
\hline \multirow[b]{2}{*}{ spiking level, $\mu \mathrm{g} / \mathrm{kg}$} & \multicolumn{2}{|c|}{ maize } & \multicolumn{2}{|c|}{ wheat } & \multicolumn{2}{|c|}{ rice } \\
\hline & recovery $(n=9, \%)$ & RSD (\%) & recovery $(n=9, \%)$ & RSD (\%) & recovery $(n=9, \%)$ & RSD (\%) \\
\hline $\operatorname{LOQ}^{a}$ & 91.2 & 4.7 & 84.7 & 8.6 & 89.6 & 6.2 \\
\hline 20 & 97.4 & 2.4 & 90.2 & 4.6 & 105.2 & 1.4 \\
\hline 40 & 94.5 & 3.7 & 96.5 & 2.2 & 101.7 & 2.7 \\
\hline 100 & 102.1 & 5.7 & 101.6 & 7.7 & 105.4 & 4.2 \\
\hline
\end{tabular}

results. And, therefore, the real mass resolution and mass accuracy were observed for each determination. The results revealed the real mass resolution of Q-Orbitrap MS proliferated greatly with the increasing level of rubrofusarin spiked in the blank matrix extracts (Figure S5). The minimum mass resolution (full-width at half-maximum, $R_{\mathrm{FWHM}}$ ) was 54000 , 50000 , and 63000 for the maize, wheat, and rice matrix, respectively. This indicated the co-eluting compounds with a mass difference less than $5.3 \mathrm{mmu}$ relative to rubrofusarin (A and $A+1$, approximately $20 \mathrm{ppm}$ for $\mathrm{m} / z$ of 272.0723 and 273.0757) would interfere with its quantitation in the matrix samples. Therefore, a mass accuracy tolerance of $20 \mathrm{ppm}$ was used to extract the ion chromatogram of $\mathrm{m} / z$ of 272.0723 and 273.0757. The results revealed that no obvious interfering components could be observed around the ion peak of rubrofusarin in these three blank matrixes (Figure S6). Furthermore, the obtained mass accuracy of rubrofusarin was within $\pm 1 \mathrm{ppm}$ for all three matrixes. These results were consistent with other studies using Q-Orbitrap MS analyzers that had been validated to provide accurate quantitative results. $^{28,29}$

The mean recoveries $(n=9)$ ranged from 84.7 to $105.4 \%$ for all three matrixes with relative standard deviation (RSD) in the range from 1.4 to $8.4 \%$ (Table 1 ). These results were in good agreement with the guideline ranges $(80-110 \%$, RSD $<10 \%)$ imposed by Commission Decision No. (EC) 2002/657. ${ }^{30}$

Quantitation of Rubrofusarin, Rubrofusarin Isomer, and Their Quinone Forms in Grains. The concentrations of rubrofusarin isomer and their quinone forms were estimated by their peak areas relative to rubrofusarin in the corresponding grain sample. Totally, 61 (29\%) maize samples, 104 (80\%) rice samples, and $70(35 \%)$ wheat samples were determined to be rubrofusarin positive, and the concentration ranged from 3.278 to $33.82 \mu \mathrm{g} / \mathrm{kg}$, from 0.815 to $61.86 \mu \mathrm{g} / \mathrm{kg}$, and from 7.362 to $47.24 \mu \mathrm{g} / \mathrm{kg}$ for the maize, rice, and wheat samples, respectively (Figure 4). Rubrofusarin isomer could only be detected in trace in rice and wheat samples and the concentration was below the LOD (not shown). The quinone forms of rubrofusarin and rubrofusarin isomer were observed to be positive only in the rice and wheat samples. The estimated concentration of quinone form of rubrofusarin isomer ranged from 0.8928 to $12.85 \mu \mathrm{g} / \mathrm{kg}$ (40 samples) and $0.2749-10.72 \mu \mathrm{g} / \mathrm{kg}$ (36 samples) for rice and wheat samples, respectively, which were relative higher than those of quinone form of rubrofusarin (Figure S7). These results indicated the predominating occurrence of rubrofusarin and quinone form of rubrofusarin isomer in the grains.

Comparison Between Rubrofusarin and the Known Mycotoxins in Grains. The concentrations of the known mycotoxins including trichothecene toxins, ZEN, fumonisins, aflatoxins, and OTA are summarized in Table S1. Fumonisins, trichothecene toxins, and aflatoxin B1 were the most prevalent mycotoxins found in maize, wheat, and rice samples,

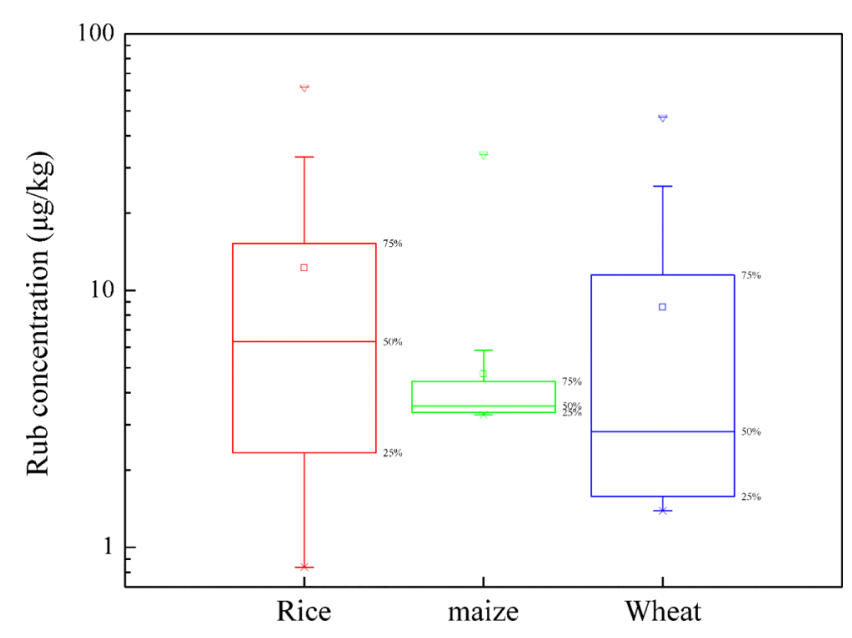

Figure 4. Concentrations of rubrofusarin in rice, wheat, and maize samples. $\nabla$ : maximum; $\square$ : average; *: minimum.

respectively. The mean concentrations of fumonisins $\mathrm{B} 1$, DON, and aflatoxin B1 were 2588, 1856, and $8.252 \mu \mathrm{g} / \mathrm{kg}$ for maize, wheat, and rice samples, respectively. Rubrofusarin, DON, and ZEN could all be produced by F. graminearum; however, the significant correlation $(p<0.05)$ between rubrofusarin and DON and ZEN could only be observed in wheat samples (Figure 5). The ratios of rubrofusarin to DON and ZEN were relatively higher in rice samples compared with those in wheat and maize samples. These results indicated that the generation of rubrofusarin might deserve more attention in rice samples. However, further investigations of high incidence of $F$. graminearum in rice were necessary to confirm this observation.

Fragmentation Studies of Rubrofusarin Using QOrbitrap MS. The Q-Orbitrap operating in $\mathrm{ddMS}^{2}$ mode provides accurate masses of product ions and therefore offers a higher level of confidence in analyte identification than a triple quadrupole mass analyzer in the tandem mass spectrometry (MS/MS) mode. The ddMS ${ }^{2}$ spectra of rubrofusarin collected in this study revealed a mass accuracy within $\pm 5 \mathrm{ppm}$ for the rubrofusarin standard, spiked blank matrix, and rubrofusarinpositive samples. The normalized collision energy setting of 60 was selected to collect the standard ddMS ${ }^{2}$ spectra, and the mass within the range of 180-273 were selected as the characteristic fragment ions of rubrofusarin. Totally, 10 fragment ions with relative higher abundance $(>5 \%, 5$ ions $>10 \%)$ were screened out (Table S2). The predicted formulas, possible structures, and generation mechanisms are illustrated in Figure 6. Fragmentation begun by the protonation of oxygen atom in rubrofusarin $(m / z 273.0757)$, and this process could generate three types of protonated products. These included the protonation of oxygen in the methoxy group (ion 1A), the phenolic hydroxy group (ion 
$\mathrm{a}$

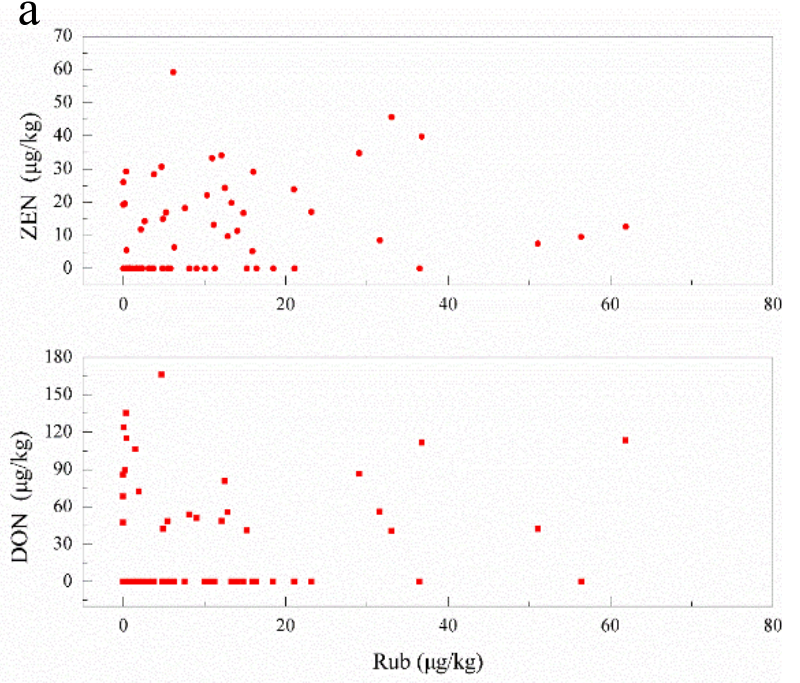

$\mathrm{b}$
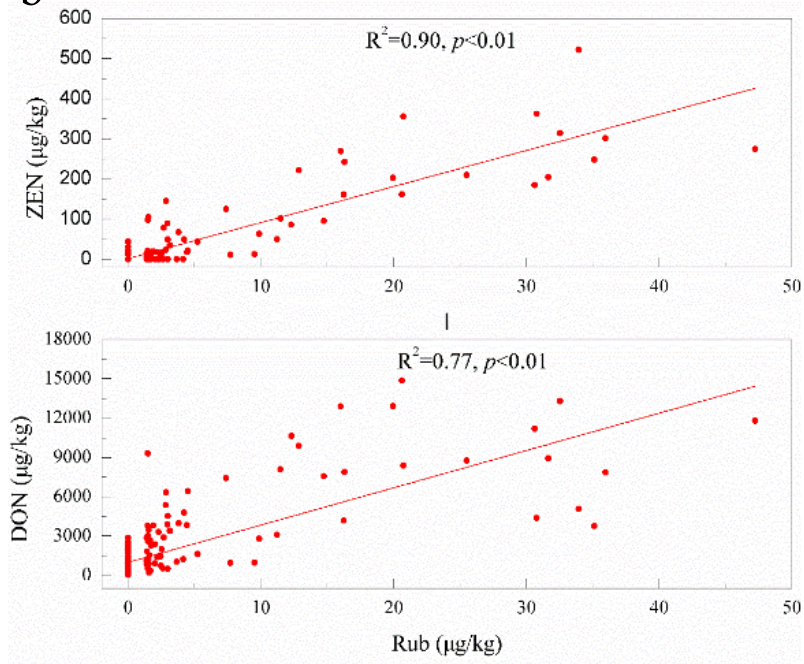

c
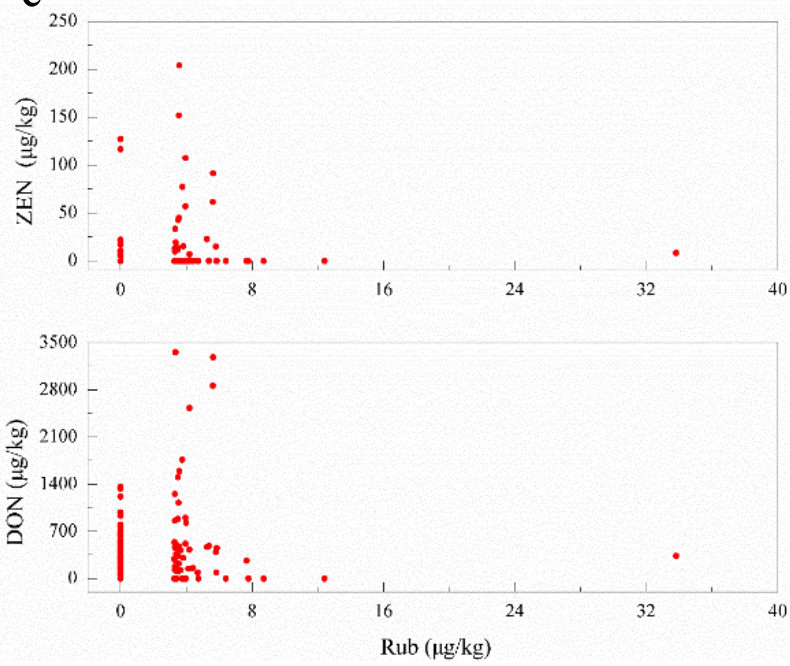

Figure 5. Correlations between rubrofusarin, DON, and ZEN in rice (a), wheat (b), and maize (c) samples, respectively. The linear regression was fitted for the wheat samples.

$2 \mathrm{~A}$ ), and the carbonyl group (ion 3A); the corresponding radical form could also be generated (ions $1 \mathrm{~B}$ and $3 \mathrm{~B}$ ). The $i$-cleavage of ions $1 \mathrm{~A}$ and $1 \mathrm{~B}$ led to the loss of methoxy group and the formation of $\left[\mathrm{C}_{14} \mathrm{H}_{11} \mathrm{O}_{4}\right]^{+}$ion $\mathrm{A}(\mathrm{m} / z$ 243.0652) and $\left[\mathrm{C}_{14} \mathrm{H}_{8} \mathrm{O}_{4}{ }^{\circ}\right]^{+}$ion $(\mathrm{m} / z 240.0417)$, respectively. $\left[\mathrm{C}_{14} \mathrm{H}_{11} \mathrm{O}_{4}\right]^{+}$ ion $\mathrm{A}$ could further undergo rearrangement and cleavage to lose phenolic hydroxy, methylene, and $\mathrm{C}_{3} \mathrm{H}_{4} \mathrm{O}$, which corresponded to the formation of $\left[\mathrm{C}_{14} \mathrm{H}_{11} \mathrm{O}_{3}\right]^{+}$ion $(m / z$ 227.0703), $\left[\mathrm{C}_{13} \mathrm{H}_{9} \mathrm{O}_{4}\right]^{+}$ion $(m / z 229.0495)$, and $\left[\mathrm{C}_{11} \mathrm{H}_{7} \mathrm{O}_{3}\right]^{+}$ion $(\mathrm{m} / z$ $187.0390)$, respectively. The $a$-cleavage of ion $1 \mathrm{~B}$ leaded to the loss of methyl group and the formation of $\left[\mathrm{C}_{14} \mathrm{H}_{10} \mathrm{O}_{5}{ }^{\bullet}\right]^{+}$ion $(\mathrm{m} /$ $z$ 258.0523). $\left[\mathrm{C}_{14} \mathrm{H}_{10} \mathrm{O}_{5}{ }^{\bullet}\right]^{+}$ion could further undergo $i$-cleavage and loss of carbonyl group to form $\left[\mathrm{C}_{13} \mathrm{H}_{10} \mathrm{O}_{4}{ }^{\bullet}\right]^{+}$ion $\mathrm{A}(\mathrm{m} / z$ 230.0574), which subsequently lost one molecular $\mathrm{H}_{2} \mathrm{O}$ to form $\left[\mathrm{C} 13 \mathrm{H} 8 \mathrm{O} 3^{\circ}\right]^{+}$ion $(\mathrm{m} / z 212.0469$, $i$-cleavage $)$. Ion $2 \mathrm{~A}$ could undergo $a$-cleavage and then re reaction to loss $\mathrm{HCO}$ and form $\left[\mathrm{C}_{14} \mathrm{H}_{11} \mathrm{O}_{4}\right]^{+}$ion B $(m / z 243.0652) .\left[\mathrm{C}_{14} \mathrm{H}_{11} \mathrm{O}_{4}\right]^{+}$ion B could further lose $\mathrm{HCO}$ and the methoxy group to form $\left[\mathrm{C}_{12} \mathrm{H}_{9} \mathrm{O}_{2}\right]^{+}$ ion $(\mathrm{m} / z$ 185.0579). The $i$-cleavage of ion $2 \mathrm{~A}$ would loss one molecular $\mathrm{H}_{2} \mathrm{O}$ to form $\left[\mathrm{C}_{15} \mathrm{H}_{11} \mathrm{O}_{4}\right]^{+}$ion $(\mathrm{m} / z$ 255.0652), which would lose the methoxy group to form $\left[\mathrm{C}_{14} \mathrm{H}_{11} \mathrm{O}_{3}\right]^{+}$ion $(\mathrm{m} / z$ 227.0703). Ion 3A would undergo either $a$-cleavage and then $r d$ reaction or $a$-cleavage and then rearrangement and $i$ cleavage to lose $\mathrm{HCO}$ and form $\left[\mathrm{C}_{14} \mathrm{H}_{11} \mathrm{O}_{4}\right]^{+}$ion $\mathrm{C}(\mathrm{m} / z$ 243.0652 ). Ion 3B could undergo either subsequent $a$-cleavage, rearrangement, and $i$-cleavage or rearrangement, $a$-cleavage, rearrangement, and $i$-cleavage to form $\left[\mathrm{C}_{13} \mathrm{H}_{10} \mathrm{O}_{4}{ }^{\bullet}\right]^{+}$ion $\mathrm{B}(\mathrm{m} / z$ $230.0574)$. These results indicated a wealth of information could be obtained from the ddMS ${ }^{2}$ spectra that allowed for advanced data interpretation to provide solid confidence for the identification of the rubrofusarin in grains.

The good mass accuracy and full mass scan by Q-Orbitrap MS were validated to be useful for the retrospective analysis of unnoticed fungal metabolites in grains, as illustrated by the identification, confirmation, and quantitation of rubrofusarin, rubrofusarin isomer, and their quinone forms in this study. These toxic metabolites were confirmed to be co-occurrence with the regulated Fusarium mycotoxins like DON and ZEN. And, therefore, the contribution of these compounds should be considered when assessing the overall toxicity of Fusarium mycotoxins in grains. However, further data including the detailed toxicity and occurrence levels in grains are necessary to evaluate the exposure risk of these compounds from diet intake.

\section{MATERIALS AND METHODS}

Chemicals and Materials. Rubrofusarin was purchased in neat form from Abcam (Shanghai, China). HPLC-grade acetonitrile and methanol were purchased from Fisher Scientific (Pittsburgh, PA). MS-grade formic acid and ammonium formate were purchased from Sigma-Aldrich (St. Louis, MO). Positiveand negative-ion solutions used to calibrate the Q-Orbitrap MS were purchased from Thermo Fisher Scientific Inc. (San Jose, CA). MilliQ water (18.2 M $\Omega$ ) was used in all solution preparation.

Standards Preparation. Stock standard solutions $(200 \mu \mathrm{g} /$ $\mathrm{mL}$ ) of rubrofusarin were prepared by dissolving $1.0 \mathrm{mg}$ of the standard in $5 \mathrm{~mL}$ of acetonitrile. A working standard solution of $2.0 \mu \mathrm{g} / \mathrm{mL}$ was prepared by diluting the stock standard solution with water/extraction solution (1:1, v:v; extraction solution consist of 70:29:1 acetonitrile/water/acetic acid, v:v:v). Stock solutions and working standard solutions were stored at $-20^{\circ} \mathrm{C}$.

Eleven standard solutions of rubrofusarin $(0.025,0.05,0.1$, $0.25,0.5,1.0,2.5,5.0,10,25,50$, and $100 \mu \mathrm{g} / \mathrm{L})$ were prepared by successive dilution of the working standard solutions with water/extraction solution or water/matrix-matched extraction solution $(1: 1, \mathrm{v}: \mathrm{v})$, respectively. 

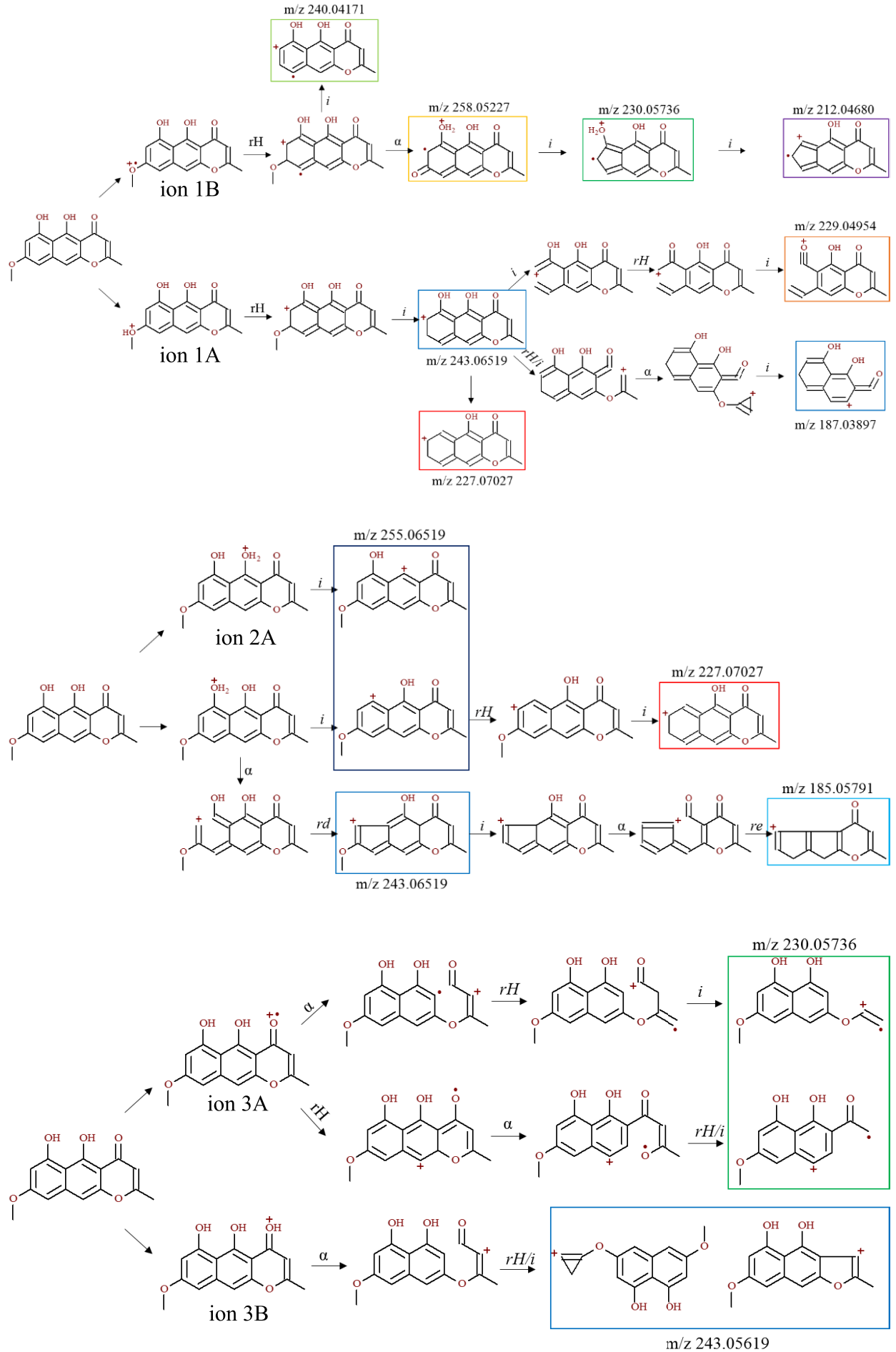

Figure 6. The proposed fragmentation mechanism of rubrofusarin. The structures indicated with square frames were the observed product ions. $a: a-$ cleavage. $i$ : $i$-cleavage. $\mathrm{rH}$ : hydrogen rearrangement. re: elimination reaction.

Sample Preparation. Maize, wheat, and rice samples were collected from Heilongjinag, Shandong, Hebei, and Sichuan province of China after grain harvest. A total of 540 samples including 210 maize, 200 wheat, and 130 rice samples were analyzed. As previously described ${ }^{31}$ bulk grains were milled and filtered through a 40 -mesh sieve. A volume of $20 \mathrm{~mL}$ of extraction solution was added to $5.00 \pm 0.05 \mathrm{~g}$ of ground sample in Nunc $50 \mathrm{~mL}$ centrifuge tubes (Thermo Fisher Scientific Inc., San Jose, CA). Samples were extracted for $30 \mathrm{~min}$ using a highspeed vortex mixer (2000 rpm, Targin, VX-III, China) and followed by centrifugation for $5 \mathrm{~min}$ at $5000 \mathrm{~g}, 25^{\circ} \mathrm{C}$. A $500 \mu \mathrm{L}$ portion of the extract was transferred to a $1.5 \mathrm{~mL}$ polyethylene tube (Corning Inc., Corning, NY), followed by the addition of $500 \mu \mathrm{L}$ water. The tube was vortexed for $15 \mathrm{~s}$ and the mixture was filtered through a $13 \mathrm{~mm} \times 0.2 \mu \mathrm{m}$ poly(tetrafluoroethylene) syringe filter (Pall Life Sciences, Ann Arbor, MI). The filtrate was directly transferred into an autosampler vial (CNW, Shanghai, China).

Recovery Studies. Three blank samples were selected for maize, wheat, and rice matrix. Spiking solution volumes of 100 
$\mu \mathrm{L}$ were added to the blank samples to achieve spiking levels of LOQ 20, 40, and $100 \mu \mathrm{g} / \mathrm{kg}$ for wheat, maize, and rice matrixes, respectively. The spiked blank samples were subject to extraction procedure as mentioned for the sample preparation described above.

Limit of Detection and Quantitation Studies. The LOD and LOQ values were determined using blank samples spiked with different concentrations of rubrofusarin. LOD and LOQ were calculated as the minimum concentration of rubrofusarin that produced chromatographic peak areas with a signal-to-noise ratio of 3 and 10, respectively.

UHPLC/Q-Orbitrap MS Analysis. A Dionex ultrahighperformance chromatograph coupled with a quadrupole orbital trap mass spectrometer (Q-Orbitrap MS, QExactive, Thermo Fisher Scientific, San Jose, CA) was employed for all the analysis. The UHPLC method was performed on a Waters CORTECSTM UPLC C18 column $(100 \mathrm{~mm} \times 2.1 \mathrm{~mm}, 1.6 \mu \mathrm{m})$ using a flow rate of $0.3 \mathrm{~mL} / \mathrm{min}$. The mobile phases were MilliQ water containing $0.1 \%$ formic acid and $0.1 \%$ ammonium formate (A) and HPLC-grade methanol containing $0.1 \%$ formic acid and $0.1 \%$ ammonium formate (B). The multistep elution procedure was performed as follows: $0-2 \mathrm{~min}, 90 \% \mathrm{~B} ; 2-3 \mathrm{~min}, 90-80 \%$ B; 3-4 min, 80-79\% B; 4-5 min, 79-74\% B; 5-7 min, 74\% B; 7-10.5 min, 74-40\% B; $10.5-16.5 \mathrm{~min}, 40-25 \% \mathrm{~B} ; 16.5-17$ $\min , 25-5 \% \mathrm{~B} ; 17-20 \mathrm{~min}, 5 \% \mathrm{~B} ; 20-21 \mathrm{~min}, 5-90 \% \mathrm{~B}$; and 21-24 min, $90 \% \mathrm{~B}$. The column temperature was set at $40{ }^{\circ} \mathrm{C}$ and the injection volume was set at $2 \mu \mathrm{L}$.

The Q-orbital trap MS was equipped with a heated electrospray ionization probe and a collision cell to carry out higher-energy collision dissociation-based tandem MS experiments $\left(\mathrm{MS}^{2}\right)$. The Q-Orbitrap MS was operated in either fullscan MS or full-scan data-dependent $\mathrm{MS}^{2}\left(\mathrm{ddMS}^{2}\right)$ mode for quantitative or qualitative analysis. The instrument was tuned and the mass accuracy was calibrated each week and any time when mass accuracy of analyte exceeded $1 \mathrm{ppm}$. The instrument and data collection parameters are listed in Table S3.

F. graminearum Cultivation. Strain of F. graminearum was previously isolated from the grain samples and stored on potato dextrose agar medium in our laboratory. For the culture on the maize powder, $1 \mathrm{~mL}$ of spore suspension (about $1 \times 10^{6}$ spores/ $\mathrm{mL}$ ) was added to $50 \mathrm{~g}$ autoclaved blank maize powder in a 250 $\mathrm{mL}$ conical flask and the moisture content was adjusted to $30 \%$ (w:w). The matrix medium was kept at $28{ }^{\circ} \mathrm{C}$ for the indicated time (totally 30 days) and then subject to solvent extraction as described above for the sample preparation after lyophilization.

Data Analysis. From the raw data, analytes were identified by their retention time $\left(t_{\mathrm{R}}\right)$ precursor ions, $\mathrm{A}+1$, and $\mathrm{A}+2$ isotopes, MS/MS mass spectra, and prediction of molecular composition from the extracted ion chromatogram using the Xcalibur Qual Browser (V.3.0 SP1.48, Thermo Fisher Scientific, San Jose, CA) with a mass accuracy $\left(\delta_{\mathrm{M}}\right)$ tolerance of $5 \mathrm{ppm}$. The $\delta_{\mathrm{M}}$ for a measured ion was calculated by dividing the difference between the theoretical and measured $m / z$ by the theoretical $\mathrm{m} / \mathrm{z}$ and expressed as part-per-million (ppm)

$$
\delta_{\mathrm{M}}=1 \times 10^{6} \times\left(\frac{m / z_{\text {measured }}-m / z_{\text {theoretical }}}{m / z_{\text {theoretical }}}\right)
$$

Screening and quantitation of the analytes were carried out using Trace finder software (Thermo Fisher Scientific, San Jose, CA). Mass Frontier (V. 7.01 SR1, Thermo Fisher Scientific, San Jose, $\mathrm{CA}$ ) software was used to predict the molecular structures in the fragmentation studies.

\section{ASSOCIATED CONTENT}

\section{Supporting Information}

The Supporting Information is available free of charge on the ACS Publications website at DOI: 10.1021/acsomega.8b02079.

Mass spectrometer and electrospray ionization parameters, the ddMS ${ }^{2}$ spectra, matrix-matched curves, mass interference in grains (PDF)

\section{AUTHOR INFORMATION}

\section{Corresponding Author}

*E-mail: wsx@chinagrain.org. Phone/Fax: +86-10-58523708.

ORCID ${ }^{\circ}$

Song-shan Wang: 0000-0001-5904-8576

Wen-bing Yin: 0000-0002-9184-3198

Notes

The authors declare no competing financial interest.

\section{ACKNOWLEDGMENTS}

This work was funded by the Government's Key Project on International Scientific and Technological Innovation Cooperation (Grant no. 2016YFE0113000), the Science and Technology project of Beijing (Z171100001317012), and the Fundamental Research Funds for the Academy of State Administration of Grain (ZX1721).

\section{REFERENCES}

(1) Figueroa, M.; Hammond-Kosack, K. E.; Solomon, P. S. A review of wheat diseases-a field perspective. Mol. Plant Pathol. 2018, 19, 15231536.

(2) Logrieco, A.; Mule, G.; Moretti, A.; Bottalico, A. Toxigenic Fusarium species and mycotoxins associated with maize ear rot in Europe. Eur. J. Plant Pathol. 2002, 108, 597-609.

(3) Matumba, L.; Sulyok, M.; Monjerezi, M.; Biswick, T.; Krska, R. Fungal metabolites diversity in maize and associated human dietary exposures relate to micro-climatic patterns in malawi. World Mycotoxin J. 2015, 8, 269-282.

(4) Zmudzki, J.; Wiśniewska-Dmytrow, H. Limits and regulations for mycotoxins in food and feed. Pol. J. Vet. Sci. 2004, 7, 211-216.

(5) van Egmond, H. P. Worldwide regulations for mycotoxins. Adv. Exp. Med. Biol. 2002, 504, 257-269.

(6) van Egmond, H. P.; Schothorst, R. C.; Jonker, M. A. Regulations relating to mycotoxins in food: perspectives in a global and European context. Anal. Bioanal. Chem. 2007, 389, 147-157.

(7) Ciasca, B.; Pascale, M.; Altieri, V. G.; Longobardi, F.; Suman, M.; Catellani, D.; Lattanzio, V. M. T. In house validation and small scale collaborative study to evaluate analytical performances of multimycotoxin screening methods based on liquid chromatography - high resolution mass spectrometry. Case study: Fusarium toxins in wheat. $J$. Mass Spectrom. 2018, 53, 742-752.

(8) Martínez-Domínguez, G.; Romero-Gonzalez, R.; Garrido Frenich, A. Multi-class methodology to determine pesticides and mycotoxins in green tea and royal jelly supplements by liquid chromatography coupled to Orbitrap high resolution mass spectrometry. Food Chem. 2016, 197, 907-915.

(9) Lattanzio, V. M.; Visconti, A.; Haidukowski, M.; Pascale, M. Identification and characterization of new Fusarium masked mycotoxins, T2 and HT2 glycosyl derivatives, in naturally contaminated wheat and oats by liquid chromatography-high-resolution mass spectrometry. J. Mass Spectrom. 2012, 47, 466-475.

(10) Walravens, J.; Mikula, H.; Rychlik, M.; Asam, S.; Ediage, E. N.; Di Mavungu, J. D.; Van Landschoot, A.; Vanhaecke, L.; De Saeger, S. Development and validation of an ultra-high-performance liquid chromatography tandem mass spectrometric method for the simultaneous determination of free and conjugated Alternaria toxins in cereal-based foodstuffs. J. Chromatogr. A 2014, 1372C, 91-101. 
(11) Anfossi, L.; Giovannoli, C.; Baggiani, C. Mycotoxin detection. Curr. Opin. Biotechnol. 2016, 37, 120-126.

(12) Selvaraj, J. N.; Wang, Y.; Zhou, L.; Zhao, Y.; Xing, F.; Dai, X.; Liu, $\mathrm{Y}$. Recent mycotoxin survey data and advanced mycotoxin detection techniques reported from China: a review. Food Addit. Contam., Part A: Chem., Anal., Control, Exposure Risk Assess. 2015, 32, 440-452.

(13) Ren, Y.; Zhang, Y.; Shao, S.; Cai, Z.; Feng, L.; Pan, H.; Wang, Z. Simultaneous determination of multi-component mycotoxin contaminants in foods and feeds by ultra-performance liquid chromatography tandem mass spectrometry. J. Chromatogr. A 2007, 1143, 48-64.

(14) Alygizakis, N. A.; Samanipour, S.; Hollender, J.; Ibanez, M.; Kaserzon, S.; Kokkali, V.; van Leerdam, J. A.; Mueller, J. F.; Pijnappels, M.; Reid, M. J.; Schymanski, E. L.; Slobodnik, J.; Thomaidis, N. S.; Thomas, K. V. Exploring the Potential of a Global Emerging Contaminant Early Warning Network through the Use of Retrospective Suspect Screening with High-Resolution Mass Spectrometry. Environ. Sci. Technol. 2018, 52, 5135-5144.

(15) León, N.; Pastor, A.; Yusa, V. Target analysis and retrospective screening of veterinary drugs, ergot alkaloids, plant toxins and other undesirable substances in feed using liquid chromatography-high resolution mass spectrometry. Talanta 2016, 149, 43-52.

(16) Kumagai, Y.; Shinkai, Y.; Miura, T.; Cho, A. K. The chemical biology of naphthoquinones and its environmental implications. Annu. Rev. Pharmacol. Toxicol. 2012, 52, 221-247.

(17) El-Halawany, A. M.; Chung, M. H.; Nakamura, N.; Ma, C. M.; Nishihara, T.; Hattori, M. Estrogenic and anti-estrogenic activities of Cassia tora phenolic constituents. Chem. Pharm. Bull. 2007, 55, 14761482.

(18) Branco, A.; Pinto, A. C.; Brazfilho, R.; Silva, E. F.; Grynberg, N. F.; Echevarria, A. Rubrofusarin, a natural polyketide as new human topoisomerase II- $\alpha$ inhibitor. Rev. Bras. Farmacogn. 2008, 18, 703-708.

(19) Vejdovszky, K.; Warth, B.; Sulyok, M.; Marko, D. Nonsynergistic cytotoxic effects of Fusarium and Alternaria toxin combinations in Caco-2 cells. Toxicol. Lett. 2016, 241, 1-8.

(20) Jarolim, K.; Wolters, K.; Woelflingseder, L.; Pahlke, G.; Beisl, J.; Puntscher, H.; Braun, D.; Sulyok, M.; Warth, B.; Marko, D. The secondary Fusarium metabolite aurofusarin induces oxidative stress, cytotoxicity and genotoxicity in human colon cells. Toxicol. Lett. 2018, 284, 170-183.

(21) Shephard, G. S.; Burger, H. M.; Gambacorta, L.; Krska, R.; Powers, S. P.; Rheeder, J. P.; Solfrizzo, M.; Sulyok, M.; Visconti, A.; Warth, B.; van der Westhuizen, L. Mycological analysis and multimycotoxins in maize from rural subsistence farmers in the former Transkei, South Africa. J. Agric. Food Chem. 2013, 61, 8232-8240.

(22) Nichea, M. J.; Palacios, S. A.; Chiacchiera, S. M.; Sulyok, M.; Krska, R.; Chulze, S. N.; Torres, A. M.; Ramirez, M. L. Presence of Multiple Mycotoxins and Other Fungal Metabolites in Native Grasses from a Wetland Ecosystem in Argentina Intended for Grazing Cattle. Toxins 2015, 7, 3309-3329.

(23) Blandino, M.; Scarpino, V.; Vanara, F.; Sulyok, M.; Krska, R.; Reyneri, A. Role of the European corn borer (Ostrinia nubilalis) on contamination of maize with 13 Fusarium mycotoxins. Food Addit. Contam., Part A: Chem., Anal., Control, Exposure Risk Assess. 2015, 32, $533-543$.

(24) Rugbjerg, P.; Naesby, M.; Mortensen, U. H.; Frandsen, R. J. Reconstruction of the biosynthetic pathway for the core fungal polyketide scaffold rubrofusarin in Saccharomyces cerevisiae. Microb. Cell Fact. 2013, 12, No. 31.

(25) Frandsen, R. J.; Nielsen, N. J.; Maolanon, N.; Sorensen, J. C.; Olsson, S.; Nielsen, J.; Giese, H. The biosynthetic pathway for aurofusarin in Fusarium graminearum reveals a close link between the naphthoquinones and naphthopyrones. Mol. Microbiol. 2006, 61, $1069-1080$.

(26) Mock, B. H.; Robbers, J. E. Biosynthesis of rubrofusarin by Fusarium graminearum. J. Pharm. Sci. 1969, 58, 1560-1562.

(27) Jin, Y. E.; Yu, W. U.; Xin, Y. Y.; Zhou, M. H.; Xie, G.; Wang, S. X. Determination of Mycotoxins in Cereals by UPLC-Quadrupole/ Orbitrap High Resolution Mass Spectrometry. J. Instrum. Anal. 2017, $36,449-456$.
(28) Ponzetto, F.; Boccard, J.; Baume, N.; Kuuranne, T.; Rudaz, S.; Saugy, M.; Nicoli, R. High-resolution mass spectrometry as an alternative detection method to tandem mass spectrometry for the analysis of endogenous steroids in serum. J. Chromatogr. B: Anal. Technol. Biomed. Life Sci. 2017, 1052, 34-42.

(29) Farré, M.; Pico, Y.; Barcelo, D. Application of ultra-high pressure liquid chromatography linear ion-trap orbitrap to qualitative and quantitative assessment of pesticide residues. J. Chromatogr. A 2014, $1328,66-79$.

(30) European Commission Commission Decision 2002/657/EC Implementing Council Directive 96/23/EC Concerning the Performance of Analytical Methods and the Interpretation of Results. OJ L221/ 8-36, 2002.

(31) Ye, J.; Wu, Y.; Guo, Q.; Lu, M.; Wang, S.; Xin, Y.; Xie, G.; Zhang, Y.; Mariappan, M.; Wang, S. Development and Interlaboratory Study of a Liquid Chromatography Tandem Mass Spectrometric Method for the Determination of Multiple Mycotoxins in Cereals Using Stable Isotope Dilution. J. AOAC Int. 2018, 101, 667-676. 